Revue de droit comparé du travail et de la sécurité sociale

\title{
La couverture sociale en France : dans le sens des courants dominants
}

Jean-Pierre Laborde

\section{(2) OpenEdition}

1 Journals

Édition électronique

URL : https://journals.openedition.org/rdctss/1689

DOI : $10.4000 /$ rdctss. 1689

ISSN : 2262-9815

Éditeur

Centre de droit comparé du travail et de la sécurité sociale

Édition imprimée

Date de publication : 1 avril 2019

Pagination : 214-217

ISSN : 2117-4350

\section{Référence électronique}

Jean-Pierre Laborde, "La couverture sociale en France : dans le sens des courants dominants »,

Revue de droit comparé du travail et de la sécurité sociale [En ligne], 1 | 2019, mis en ligne le 01 novembre 2021, consulté le 13 novembre 2021. URL : http://journals.openedition.org/rdctss/1689 ; DOI : https:// doi.org/10.4000/rdctss. 1689

\section{(c) $(7)(9$}

Revue de droit comparé du travail et de la sécurité sociale est mise à disposition selon les termes de la Licence Creative Commons Attribution - Pas d'Utilisation Commerciale - Pas de Modification 4.0 International. 


\title{
JEAN-PIERRE LABORDE
}

\author{
UNIVERSITÉ DE BORDEAUX
}

\section{LA COUVERTURE SOCIALE EN FRANCE : DANS LE SENS DES COURANTS DOMINANTS}

Il est assez difficile, dans une veille de droit de la sécurité sociale et de la couverture sociale qui se veut aussi large que possible, de ne pas tenir compte de la grande diversité de la couverture du système français de protection sociale, qui comprend en effet non seulement la Sécurité sociale proprement dite, mettant en œuvre pour l'essentiel des dispositifs contributifs, mais aussi la protection sociale complémentaire, qui vient en quelque sorte s'ajouter à la Sécurité sociale de base, mais encore l'aide et l'action sociales, toutes deux constituées de prestations non contributives, et même l'indemnisation du chômage, dans ses deux versants, assurantiel et assistantiel. La difficulté est redoublée quand la période considérée, en l'occurrence le second semestre de l'année 2018, se situe entre les grandes réformes initiales du quinquennat du Président Macron, commencé en 2017, et la nouvelle et ambitieuse réforme, que l'on annonce pour relativement bientôt, du système des retraites.

Pour autant, il serait sans doute erroné de considérer que la période à l'étude n'aurait pas vu s'affirmer encore davantage les courants dominants dans l'évolution contemporaine de notre protection sociale et, à l'intérieur de celle-ci, de notre Sécurité sociale, poussant à des remaniements importants de la couverture en même temps qu'à la fiscalisation progressive de son financement.

Au premier titre, nous citerons bien sûr les dispositions de la loi sur la liberté de choisir son avenir professionnel du 5 septembre 2018, qui, il est vrai avec prudence, ouvre l'indemnisation du chômage aux salariés démissionnaires et aux travailleurs indépendants. Ce n'est pas tout à fait une surprise puisque la loi du 5 septembre 2018 reprend ici, pour l'essentiel, un accord national interprofessionnel du 22 février 2018, déjà fort complètement présenté dans cette Revue. ${ }^{1}$ II n'en reste pas moins que, même s'il est encore trop tôt pour discerner une réelle universalisation de l'assurance chômage, une tendance paraît bien se dessiner en ce sens. ${ }^{2}$ Plus largement, il convient de se pencher sur les évolutions touchant aussi bien la sécurité sociale (I) que la protection sociale (II).

I. Les tendances générales de l'évolution de la sécurité sociale (1) ne doivent pas faire perdre de vue l'évolution qui touche aussi le contentieux du recouvrement des cotisations et contributions (2).

1. Même si elles ne sont pas nécessairement spectaculaires, les dispositions de la loi $n^{\circ}$ 2018-1203 du 22 décembre 2018 de financement de la sécurité sociale pour 2019 sont révélatrices, fût-ce indirectement, des tendances de fond qui orientent progressivement

1 M. Badel, «Indemnisation du chômage : la réforme en marche », Revue du droit comparé du travail et de la sécurité sociale 2018/1, p. 160.

2 Cf. Th. Montpellier, "Vers une indemnisation du chômage plus universelle? ", JCP Social 2018, n 1313, p. 23. 
notre système de sécurité sociale vers un modèle qui pourrait être fort différent du modèle socioprofessionnel d'origine. Ainsi les objectifs majeurs pour l'exercice à venir sont-ils d'encourager le travail, principalement par la baisse des cotisations et des coûts salariés, de protéger les plus vulnérables, notamment par un ciblage plus grand des revalorisations de prestations sur les personnes les plus défavorisées, et d'engager la transformation du système de santé. Même si on laisse de côté le troisième objectif, nullement négligeable par ailleurs, les deux premiers prolongent une évolution du financement vers une fiscalisation toujours plus importante et une évolution des prestations vers une attention toujours plus grande aux ressources dont disposent ou non les bénéficiaires.

La propension à fiscaliser une couverture particulièrement attentive aux catégories sociales de faibles ressources n'a pas encore pris une telle proportion qu'elle transforme radicalement la nature de notre système de sécurité sociale. A supposer qu'une telle transformation soit souhaitable, il y faudrait des mesures beaucoup plus tranchées. Pour autant, les circonstances actuelles, particulièrement difficiles sur le terrain social avec le mouvement dit des Gilets Jaunes, paraissent accentuer ces tendances déjà repérées. Ainsi la loi du 24 décembre 2018, portant mesures d'urgence économiques et sociales, encourage les employeurs des secteurs privé comme public à verser entre le 11 décembre 2018 et le 31 mars 2019 une prime qui, dans la limite de 1000 euros, sera exonérée de tout impôt, cotisation et contribution, dès lors que la rémunération du bénéficiaire est inférieure à trois fois la valeur du Smic. On retrouve ici la tendance à l'exonération de cotisations comme le ciblage des dispositifs favorable sur des catégories, ici de salariés, caractérisées par la modicité de leurs revenus ou de leurs ressources. Parallèlement, la même loi avance

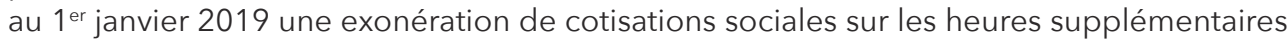
qui était seulement prévue, dans le premier état de la loi de financement de la sécurité sociale pour 2019, pour le $1^{\text {er }}$ septembre 2019.

S'agissant du renforcement du ciblage des prestations, la loi de financement de la sécurité sociale tend à dissocier du taux de l'inflation le taux de revalorisation des prestations qui, pour nombre d'entre elles, est limité à $0.3 \%$, tout en maintenant un taux de revalorisation lié à celui de l'inflation et donc a priori plus important pour ce que l'on appelle les minima sociaux, notamment les prestations qui composent le minimum vieillesse, l'allocation veuvage et aussi le revenu de solidarité active.

2. La loi $n^{\circ}$ 2018-727 du 10 août 2018 pour un Etat au service d'une société de confiance introduit l'idée d'un droit à l'erreur dans les relations entre les administrés et les services publics. La Sécurité sociale gérant une mission de service public, ce droit à l'erreur devrait donc désormais être reconnu aux entreprises cotisantes dans leurs relations avec les Urssaf, qui recouvrent notamment les cotisations patronales et salariales. Les premiers commentateurs paraissent cependant douter de l'effectivité de ce nouveau droit.

Pourra-t-on attendre davantage de l'extension au contentieux du recouvrement des cotisations de la procédure de médiation, par laquelle les litigants confient à un tiers impartial le soin de rapprocher leurs points de vue, par un avis qui ne les lie pas mais peut leur éviter le contentieux? Peut-être. L'article L. 217-7-I-1 nouveau du Code de la sécurité sociale énonce en tout cas, dans la rédaction que lui donne l'article 34 de la loi du 10 août 2018, que « les réclamations concernant les relations entre un organisme de sécurité sociale relevant du présent livre et ses usagers peuvent être présentées, sans préjudice des voies de recours existantes, devant le médiateur de l'organisme concerné ». II ajoute que «Le médiateur est désigné par le directeur de lıorganisme. II exerce ses fonctions en toute impartialité et dans le respect de la confidentialité des informations 
dont il a à connaître. Et il précise qu'«il formule auprès du directeur ou des services de l'organisme des recommandations pour le traitement de ces réclamations, dans le respect des dispositions législatives et réglementaires en vigueur ».

Sans doute ne faut-il pas s'exagérer la portée effective d'une procédure de médiation qui restera sans doute relativement marginale. Pour autant, elle montre bien l'importance donnée à tout ce qui peut permettre de réduire les contentieux en procéduralisant en quelque sorte l'énoncé du différend et les moyens alternatifs de le régler.

II. Le 13 septembre 2018, le Président Macron a fait connaître un Plan Pauvreté, de prévention et de lutte contre la pauvreté, qui affecte un engagement de huit milliards d'euros sur les quatre ans à venir au service d'un objectif ambitieux, celui d'empêcher la reproduction de la pauvreté, d'éradiquer la précarité, et de fonder la protection sociale du XXlème siècle. Rien de moins. En tout cas, le Plan couvre cinq grands champs, la petite enfance, l'enfance, les jeunes, l'accompagnement vers l'emploi et les minima sociaux. A l'énoncé de ces domaines, on ne peut manquer d'être frappé par l'accent mis sur la situation des plus jeunes. Il s'agit en effet d'éviter qu'un enfant né pauvre reste à l'âge adulte dans la pauvreté et, dans cette perspective, il convient d'agir dès la petite enfance, en élargissant l'accueil dans les crèches et par les assistantes maternelles des enfants dont les familles sont en difficulté. Il faut aussi, par la suite, lutter contre le décrochage scolaire de certains mineurs et contre le chômage des primo-demandeurs d'emploi.

Concernant précisément l'accompagnement vers l'emploi, c'est un service public nouveau de l'insertion qui serait créé. S'agissant des minima sociaux, le Plan annonce la perspective d'un revenu universel d'activité, qui se substituerait aux minima sociaux actuels ou au moins à certains d'entre eux. II s'agirait donc, à la différence du Revenu universel proposé par Monsieur Benoît Hamon, le candidat socialiste à l'élection présidentielle de 2017, d'un revenu subordonné à une condition de ressources. Comme en revanche il en a toujours été depuis la création du revenu minimum d'insertion en 1988, ce revenu s'accompagnerait d'un dispositif contractuel, énergiquement tourné vers la recherche et l'obtention d'un emploi. Comme, pour l'heure et depuis maintenant trois décennies, les résultats sur ce terrain sont fort limités, on peut donc penser que le nouveau revenu universel d'activité relèverait davantage d'une simplification de dispositifs aujourd'hui assez complexes que d'une transformation en profondeur de ce type de prestations, étant ajouté que l'appellation de revenu universel ne serait vraiment justifiée que si ce revenu était alloué sans condition à chacun. On en reste fort loin.

Plus généralement, l'Association des cadres territoriaux de l'action sociale (Actas) regrette 3 que les personnes en situation de handicap, migrants, mineurs non accompagnés, personnes âgées, soient peu touchées par les différents versants du plan et en forment une sorte $d^{\prime}$ '« impensé ». Pareillement, des mesures pour le logement et l'hébergement font défaut alors qu'il s'agit de "prémices indispensables pour envisager une sortie de la pauvreté de manière durable». Rien non plus de suffisamment précis sur le rôle et les moyens des départements et des communes.

3 Actualités Sociales Hebdomadaires, n 3083, 9 novembre 2018, p. 10. 


\section{FRANCE}

Pour autant, en dépit de ses limites et de ses manques, on peut s'étonner que la publication de ce plan pauvreté n'ait pas eu toute la résonance souhaitable. Bien au contraire, le mouvement dit des Gilets Jaunes, survenu après cette annonce et sans grand lien sans doute avec elle, semble avoir concerné bien davantage les travailleurs, indépendants tout autant que salariés de ressources modestes à moyennes, que les personnes en situation de pauvreté manifeste, et il s'est préoccupé davantage des mesures fiscales, jugées excessives, que des mesures proprement sociales. 